\title{
BLOX: the Bonn lensing, optical, and X-ray selected galaxy clusters
}

\section{Cluster catalog construction $\star, \star \star$}

\author{
J. P. Dietrich ${ }^{1,2}$, T. Erben ${ }^{1}$, G. Lamer ${ }^{3}$, P. Schneider ${ }^{1}$, A. Schwope ${ }^{3}$, J. Hartlap ${ }^{1}$, and M. Maturi ${ }^{4}$ \\ 1 Argelander-Institut für Astronomie, University of Bonn, Auf dem Hügel 71, 53121 Bonn, Germany \\ e-mail: jdietric@eso.org \\ 2 ESO, Karl-Schwarzschild-Str. 2, 85748 Garching b. München, Germany \\ Astrophysikalisches Institut Potsdam, An der Sternwarte 14, 14482 Potsdam, Germany \\ ${ }^{4}$ Institut für Theoretische Astrophysik, Albert-Ueberle-Str. 2, 69120 Heidelberg, Germany
}

Received 12 February 2007 / Accepted 19 April 2007

\begin{abstract}
The mass function of galaxy clusters is an important cosmological probe. Differences in the selection method could potentially lead to biases when determining the mass function. From the optical and X-ray data of the XMM-Newton Follow-Up Survey, we obtained a sample of galaxy cluster candidates using weak gravitational lensing, the optical Postman matched filter method, and a search for extended X-ray sources. We developed our weak-lensing search criteria by testing the performance of the aperture mass statistic on realistic ray-tracing simulations matching our survey parameters and by comparing two filter functions. We find that the dominant noise source for our survey is shape noise at almost all significance levels and that spurious cluster detections due to projections of large-scale structures are negligible, except possibly for highly significantly detected peaks. Our full cluster catalog has 155 cluster candidates, 116 found with the Postman matched filter, 59 extended X-ray sources, and 31 shear selected potential clusters. Most of these cluster candidates were not previously known. The present catalog will be a solid foundation for studying possible selection effects in either method.
\end{abstract}

Key words. gravitational lensing - galaxies: clusters: general - X-rays: galaxies: clusters - catalogs - surveys

\section{Introduction}

Because the dynamical or evolutionary timescale of clusters of galaxies is not much shorter than the Hubble time, they retain a "memory" of the initial conditions for structure formation (e.g., Borgani \& Guzzo 2001). The population of clusters evolves with redshift, and this evolution depends on the cosmological model (e.g., Eke et al. 1996); therefore, the redshift dependence of the cluster abundance has been used as a cosmological test (e.g., Vikhlinin et al. 2003; Henry 2004). The dependence of the cluster abundance on cosmological parameters can be obtained either from analytical models (Press \& Schechter 1974) or, more reliably, from $N$-body simulations (e.g., Jenkins et al. 2001). What such models do predict is the abundance of dark matter halos as a function of redshift and mass.

Clusters can be selected by various methods: optical, X-ray emission, weak lensing (e.g., Schneider 1996), and - using future surveys - the Sunyaev-Zeldovich effect (SZE). Optical, $\mathrm{X}$-ray, and SZE selection of clusters depends on the baryonic

\footnotetext{
* Based on observations carried out at the European Southern Observatory, La Silla, Chile under program Nos. 170.A-0789, 70.A-0529, 71.A-0110, 072.A-0061, 073.A-0050.

$\star \star$ The cluster catalogs are only available in electronic form at the CDS via anonymous ftp to

cdsarc.u-strasbg.fr $(130.79 .128 .5)$ or via

http://cdsweb.u-strasbg/cgi-bin/qcat?]/A+A/470/821
}

content of clusters, which - compared to the predicted dark matter density - is a minor fraction of the clusters' constituents. Optical selection depends on the star formation history, and X-ray detection requires a hot intracluster medium (ICM). Weinberg \& Kamionkowski (2002) predict that up to $20 \%$ of all weak lensing clusters have not heated their ICM to a level detectable with current X-ray missions. Searching for clusters with SZE is a very promising method but has yet to produce first samples.

Common to all methods except X-ray selection is that they are prone to projections along the line-of-sight. Spectroscopy is an essential tool in distinguishing real clusters from chance projections on the sky. X-ray emission, which depends on the square of the local gas density, is not easily affected by line-of-sight projections but is susceptible to other sorts of biases, e.g., heating of the ICM in mergers.

All four selection methods are sensitive in different redshift regimes. Optical and X-ray searches depend on the luminosity distance of clusters. SZE is nearly redshift independent. Weak lensing surveys typically cover the redshift range of $0.15-0.7$. Clearly, no cluster selection method is ideal, and understanding their biases and limitations is important for precision cosmology using clusters.

Several galaxy clusters have already been found using weak gravitational lensing in recent years: in the FORS1 cosmic shear survey (Maoli et al. 2001; Hetterscheidt et al. 2005), one mass 
peak clearly coincides with an overdensity of galaxies. Dietrich et al. (2005) found a cluster in the background of the supercluster system A 222/223. Wittman et al. (2006), have published 6 new clusters detected with weak lensing from their Deep Lens Survey. These examples clearly demonstrate that this method of cluster detection in fact works.

The selection of clusters of galaxies by weak lensing, however, also faces significant methodological challenges. Even before the first spectroscopically confirmed weak-lensing detected cluster was reported (Wittman et al. 2001), Erben et al. (2000) reported a highly significant tangential alignment of galaxies around an empty spot on the sky (see also von der Linden et al. 2006). Two more of these dark clumps have been reported in the literature (Umetsu \& Futamase 2000; Miralles et al. 2002; but see also Erben et al. 2003). Recently Schirmer et al. (2007) have published a catalog of 158 shear selected peaks, 72 of them associated with bright galaxy concentrations. Of course, the reality of these dark clump detections has to be considered with caution, given that even one of them would have a profound impact on our understanding of the evolution of dark and baryonic matter in the Universe.

Several theoretical studies have recently shed some light on the problem of dark clump detection in weak lensing surveys. Among them are Hamana et al. (2004, H04) and Hennawi \& Spergel (2005, HS05) who have both used ray-tracing simulations through $N$-body simulations to study the efficiency, also called purity by other authors, and completeness of the detection of clusters of galaxies in weak lensing surveys. An important result of HS05 is that the efficiency, even in the limiting case of no intrinsic galaxy ellipticity, does not increase beyond $85 \%$. The remaining $15 \%$ of shear selected peaks are due to projections of the large-scale structure along the line of sight and will be seen as dark clumps. These could very well account for the dark clumps studied so far in detail in the literature. The efficiency naturally drops further if more realistic noise caused by the ellipticity of the background galaxies is assumed. The completeness was studied in more depth by H04, who find that, even with low significance thresholds in the selection process shear, selected samples will be incomplete, except at the highest masses.

In this paper we describe a search for galaxy clusters in the public XMM-Newton Follow-Up Survey (Dietrich et al. 2006) and our private extension to the survey. We combine the results of three independent selection methods using the aperture mass statistic (Schneider 1996) for weak lensing selection, the optical matched filter algorithm (Postman et al. 1996, hereinafter P96) for optical selection of galaxy clusters, and a search for extended $\mathrm{X}$-ray emission in the XMM-Newton data on our survey fields. The combination allows us to dig deeper into the mass function than we could do with weak lensing selection alone.

This paper is organized as follows: Sect. 2 briefly describes the XMM-Newton Follow-Up Survey, the optical data, and their reduction. Section 3 gives an overview of the X-ray data, their reduction, and then describes how we detected galaxy cluster candidates as extended X-ray sources. In Sect. 4 we present our implementation of the matched filter technique of P96 and the resulting catalog of cluster candidates. We briefly summarize the aperture mass methods (Schneider 1996) in Sect. 5 and develop our selection criteria used for cluster detection later in that section based on realistic ray-tracing simulations. We discuss and summarize our findings in Sect. 6. The catalogs of cluster candidates are available in electronic format at the CDS.

Throughout this work we assume an $\Omega_{\mathrm{m}}=0.3, \Omega_{\Lambda}=0.7$, $H_{0}=70 h_{70} \mathrm{~km} \mathrm{~s}^{-1}$ cosmology and use standard lensing notation (e.g., Bartelmann \& Schneider 2001).

\section{The XMM-Newton Follow-Up Survey}

The XMM-Newton Follow-Up Survey (XFS) consists of two parts, a public and a private one. Both were conducted with the Wide-field Imager (WFI) at the ESO/MPG-2.2 m telescope on La Silla, Chile. The survey provides optical imaging on fields for which deep, public XMM-Newton exposures exist.

\subsection{Public and private survey}

The public survey (ESO Program Id. 170.A-0789, P.I. J. Krautter as chairman of the ESO working group for public surveys) was carried out in the framework of the ESO Imaging Survey (EIS) as a collaboration between ESO, the XMM-Newton Survey Science Centre (SSC), and a group at the Institut für Astrophysik und Extraterrestrische Forschung (IAEF) at the University of Bonn. The aim of the public survey, whose observations were concluded in November 2005, was to provide optical counterparts to serendipitously detected X-ray sources in the southern hemisphere. To provide data for a minimum spectral discrimination and photometric redshift estimates, the public survey was carried out in the B-, V-, R-, and I-passbands down to a limiting magnitude of 25 mag in the AB system in all bands. All data of the public survey taken before October 16, 2003 were reduced, calibrated, and publically released in July 2005 (Dietrich et al. 2006). The total public survey comprises 15 WFI fields. Out of these, 4 are galactic fields and thus unsuitable for galaxy cluster searches. One field is a mispointing without X-ray data and high galactic absorption. The remaining 10 fields cover about $\sim 2.8$ sq. deg in BVRI.

The private extension of the survey (Program Ids. 70.A0529, 71.A-0110, 072.A-0061, 073.A-0050, P.I. P. Schneider) with the goal of a weak lensing search for galaxy clusters has been conducted as a collaboration between the IAEF and the Astrophysikalisches Institut Potsdam (AIP). Originally targeted to observe 17 additional fields in B- and R-band and to be finalized by October 2003, observations were concluded only in September 2006 due to weather and scheduling problems. Out of the 14 additional fields observed in R-band, our primary band for the lensing analysis, 13 are used for this work. This corresponds to all data obtained until September 30, 2005. B-band observations, proposed to allow for a red-sequence cluster search (Gladders \& Yee 2000), are available for 9 of these fields.

A cluster survey carried out on these fields is obviously not an unbiased survey since it includes 5 XMM-Newton observations that targeted known galaxy clusters. The impact on the total number of clusters detected, however, is small, at least for the $\mathrm{X}$-ray and optically detected clusters. The influence on the weak lensing sample is greater because weak lensing requires clusters to be fairly massive to be detectable with $2 \mathrm{~m}$ class telescopes. Previously known clusters are likely to be relatively massive and influence the smaller sample of weak lensing detected clusters more strongly.

\subsection{Optical data reduction}

We reduced the optical XFS data using the publically available GaBoDS pipeline (Erben et al. 2005) with the Guide Star Catalog version 2.2 (GSC-2.2) as the astrometric reference and the Stetson (2000) catalog for photometric calibration. A subset of our reduced data was compared against the publically released XFS data (Dietrich et al. 2006) and found to be in very good agreement with this independent reduction. 
Table 1. Effective area and number density of galaxies of WFI fields in the XFS used in the cluster search.

\begin{tabular}{|c|c|c|c|c|c|c|c|}
\hline Field & $\begin{array}{c}\alpha \\
(\mathrm{J} 2000.0)\end{array}$ & $\begin{array}{c}\delta \\
(\mathrm{J} 2000.0)\end{array}$ & $\begin{array}{c}m_{\lim } \\
(\mathrm{mag})\end{array}$ & $\begin{array}{l}\text { Seeing } \\
(\operatorname{arcsec})\end{array}$ & $\begin{array}{c}\text { Area } \\
\left(\operatorname{arcmin}^{2}\right)\end{array}$ & $\begin{array}{c}\text { Number density (WL) } \\
\left(\operatorname{arcmin}^{-2}\right)\end{array}$ & $\begin{array}{c}\text { Number density (MF) } \\
\left(\operatorname{arcmin}^{-2}\right)\end{array}$ \\
\hline BPM 16274 & $00: 50: 03.2$ & $-52: 08: 17$ & 25.38 & 0.88 & 1011 & 15.6 & 21.6 \\
\hline QSO B0130-403 & 01:33:01.9 & $-40: 06: 28$ & 24.94 & 1.01 & 925 & 7.8 & 12.7 \\
\hline CFRS 3h & 03:02:39.2 & $+00: 07: 31$ & 25.46 & 0.85 & 965 & 12.1 & 17.0 \\
\hline RX J0505.3-2849 & 05:05:20.0 & $-28: 49: 05$ & 25.58 & 0.83 & 981 & 18.2 & 25.9 \\
\hline RBS 0864-N & $10: 21: 03.8$ & $+04: 26: 23$ & 25.53 & 1.01 & 948 & 13.0 & 20.2 \\
\hline QSO B1033-033 & $10: 36: 23.7$ & $-03: 43: 20$ & 25.59 & 0.78 & 1037 & 16.8 & 22.0 \\
\hline SDSS J104433.04-012502.2 & $10: 44: 33.0$ & $-01: 25: 02$ & 25.44 & 0.73 & 997 & 16.9 & 22.6 \\
\hline MS 1054.4-0321 & $10: 56: 60.0$ & $-03: 37: 27$ & 25.35 & 0.69 & 1022 & 17.7 & 23.5 \\
\hline HE 1104-1805 & $11: 06: 33.0$ & $-18: 21: 24$ & 25.47 & 0.83 & 1030 & 13.4 & 22.4 \\
\hline PG 1115+080 & $11: 18: 17.0$ & $+07: 45: 59$ & 25.48 & 0.92 & 1051 & 12.3 & 19.6 \\
\hline CD -3307795 & $11: 29: 27.2$ & $-34: 19: 55$ & 25.04 & 0.91 & 927 & 6.1 & 19.1 \\
\hline T Leo & 11:38:27.1 & $+03: 22: 10$ & 25.47 & 0.74 & 1005 & 18.6 & 23.9 \\
\hline IRAS $12112+0305$ & $12: 13: 46.1$ & $+02: 48: 41$ & 25.51 & 0.88 & 1028 & 13.9 & 21.9 \\
\hline LBQS $1228+1116$ & $12: 30: 54.1$ & $+11: 00: 11$ & 25.09 & 1.01 & 1014 & 8.4 & 14.3 \\
\hline NGC 4666 & $12: 45: 08.9$ & $-00: 27: 38$ & 25.43 & 0.87 & 1074 & 10.7 & 17.8 \\
\hline QSO B1246-057 & $12: 49: 13.9$ & $-05: 59: 19$ & 25.21 & 0.80 & 869 & 13.0 & 19.6 \\
\hline Field 864-1 & $13: 41: 22.4$ & $+00: 23: 52$ & 25.45 & 0.88 & 1058 & 16.8 & 23.9 \\
\hline Field 864-9 & $13: 44: 36.0$ & $-00: 24: 00$ & 25.56 & 0.84 & 1045 & 16.9 & 25.6 \\
\hline A 1882 & $14: 14: 39.9$ & $-00: 19: 07$ & 25.73 & 0.72 & 1019 & 18.6 & 29.5 \\
\hline MKW 9 & $15: 32: 29.3$ & $+04: 40: 54$ & 25.56 & 0.91 & 1032 & 10.7 & 19.6 \\
\hline LBQS 2212-1759 & $22: 15: 31.7$ & $-17: 44: 05$ & 25.52 & 0.99 & 970 & 12.6 & 19.9 \\
\hline NGC 7252 & $22: 20: 44.8$ & $-24: 40: 42$ & 25.57 & 0.70 & 997 & 20.3 & 26.4 \\
\hline PHL 5200 & $22: 28: 30.4$ & $-05: 18: 55$ & 24.92 & 1.06 & 1068 & 7.8 & 11.7 \\
\hline Total/average & & & & & 23073 & 14.1 & 20.9 \\
\hline
\end{tabular}

We would like to point out that we applied the fringing removal procedure to all our R-band images to remove the low level fringing present in WFI R-band. This is a difference to the data released by Dietrich et al. (2006). Weight images describing the relative noise properties of each pixel were created to mask bad pixels or columns, cosmic rays, and other image defects masked manually, like satellite tracks and ghost images due to internal reflections.

Table 1 lists all coadded R-band images with center coordinates of the survey fields used in the present work, their $5 \sigma$ limiting magnitude computed in the Vega system in a $2^{\prime \prime}$ diameter aperture, their seeing, as well as the effective (unmasked) area used for catalog creation (see Sect. 4.1), and the number densities of the resulting lensing (see Sect. 5.2) and matched filter (Sect. 4.2) catalogs.

Two independent observations of the field RBS 0864 are used in the XFS. The WFI observations in the V- and R-bands done by Schindler et al. (RBS 0864-N in Table 1) are centered on coordinates slightly north west of the galaxy cluster and were taken with a seeing constraint of $1^{\prime \prime} .2$. The re-observation in the B- and R-bands two years later in the course of the XFS (RBS 0864-S) is centered on the cluster and was observed with the seeing constraint of $1^{\prime \prime}$. 0 of this survey. However, most data was taken well outside the specified constraint. Consequently, we used only the RBS 0864-N pointing for our weak lensing analysis. We will describe the full XFS data set including BVI passband data in a forthcoming paper (Dietrich et al., in preparation).

\section{X-ray detection}

The X-ray luminosity of the hot intracluster gas scales with the square of its density. Thus, X-ray detections of clusters of galaxies are relatively insensitive to projection effects. The high sensitivity of XMM-Newton allows us to find clusters out to very high redshifts. Two of the three most distant and spectroscopically confirmed clusters were found serendipitously with XMM-Newton. These are at redshift $z=1.39$ (Mullis et al. 2005) and $z=1.45$ (Stanford et al. 2006), the latter located in the public XFS field LBQS 2212-1759.

\subsection{X-ray data reduction}

The archival XMM-Newton data of the XFS were reduced with the latest version of the Science Analysis System available at that time (SAS-6.5.0). We briefly describe the standard processing steps employed to create X-ray source lists from the observation data files. Appendix A gives an overview of all X-ray observations considered in the present work. Some fields were observed more than once. Their data reduction requires additional steps described at the end of this section.

SAS was used to generate calibrated event lists, exposure maps describing the spatial variations of the instruments' sensitivity, and images in five energy bands ranging from 0.1-0.5 keV, 0.5-1.0 keV, 1.0-2.0 keV, 2.0-4.5 keV, and 4.5-12.0 keV. We used the standard SAS flags \#XMMEA_EM and \#XMMEA_EP to filter the event lists when generating the images. For the PN detector we imposed additional restrictions on the allowed charge patterns. For the two lowest energy bands we accepted only single events; for the three highest energy bands only events not depositing charge in more than two neighboring pixels were kept. We also excluded the energy range of $7.8-8.2 \mathrm{keV}$ from the PN data to avoid the complex of $\mathrm{Ni}-\mathrm{K} \alpha$, $\mathrm{Cu}-\mathrm{K} \alpha$, and $\mathrm{Zn}-\mathrm{K} \alpha$ fluorescence lines of the detector and surrounding structure.

Using the 15 images generated with evselect (5 images per camera and 3 cameras), the mask images, and the vignetted exposure maps, eboxdetect in local mode was run to generate a first source list. The minimum likelihood for a detection in this step was set to 5 . The sources were excised from the image by esplinemap to model the background of the images. The task esplinemap allows to describe the background either by a 
two-component model (vignetted astrophysical and unvignetted particle background) based on ray-tracing of the instruments or by a 2-d spline with a user-defined number of nodes. For the XFS the decision about which approach to use was based on visual inspection of the data.

With these background maps, eboxdetect was run in map mode to create another source list. The minimum likelihood for source detection was set to 4 in this step. This list was given together with the science images, masks, and background maps to emldetect, which performs a simultaneous maximum likelihood multi-source PSF fitting in all energy bands. The free parameters emldetect fits are the source position, source extent (core radius of a $\beta$-model), and source count rate in each energy band and instrument. Derived parameters are the total source count rate, total likelihood of detection and likelihood of detection per energy band, likelihood of source extent, and four hardness ratios between the input energy bands. In our reduction we let emldetect fit up to two sources to one source position reported by eboxdetect. The minimum likelihood for a detection was set to 6 , and the minimum extent likelihood for a source to be considered as an extended source to 4 .

It should be pointed out that the result of the PSF fitting performed by emldetect cannot be better than the available PSF models. Especially at large off-axis distances the EPIC PSF is not very well known.

For the two deepest combined fields (LBQS 2212-1759, $250 \mathrm{ks}, \mathrm{PG} 1115+080,220 \mathrm{ks}$ ) the source detection process was slightly modified with respect to the other observations. In deep observations small inaccuracies of the background fit can lead to many spurious detections of extended sources. Since in the softest band $(0.2-0.5 \mathrm{keV})$ both the MOS and PN cameras show spatial variations of the detector background and since the hardest band (4.5-12 keV) is strongly dominated by background, only the 3 bands in the range $0.5-4.5 \mathrm{keV}$ were used for source detection in the two very deep observations.

The source positions in the emldetect catalog have statistical errors on the order of $1^{\prime \prime}-2^{\prime \prime}$, plus a systematic error due to an uncertainty in the attitude of the spacecraft, which has the same size. The latter can be corrected by cross-correlating the $\mathrm{X}$-ray source positions with the more accurate positions of optical sources. The task eposcorr was used to carry out this crosscorrelation with the R-band catalogs on all fields. The production of the optical catalogs is described in detail in Sect. 4.1.

Areas of the EPIC field-of-view that are dominated by bright extended emission of the XMM-Newton target were excluded from the survey by masking out a circular area around the target before starting the detection process.

Multiple observations of one field are in general taken at different roll angles and - since the center of rotation is not the center of the FOV - different positions. The first step in combining observations is thus to bring them all to a common nominal position. This was done with the SAS task attcalc, which computes sky coordinates for event files. From these new event lists FITS images, exposure maps, and masks were created for the individual observations as described above. Science images and exposure maps were coadded weighted by the masks of the respective observation. The masks themselves are combined with logical or. The source extraction continues on the combined images, exposures maps, and masks as described above.

\subsection{Catalog of extended $X$-ray sources}

Catalogs of cluster candidates were generated from the emldetect source list. Sources with a detection of likelihood
$>15$ and extent likelihood $>4$ were considered as potential cluster candidates. Any remaining large-scale inhomogeneities in the background are sometimes detected as spurious sources and the best-fit model of emldetect is often one whose extent reaches the maximum value of 20 pixels $\left(80^{\prime \prime}\right)$, hence only sources with an extent $<20$ pixels were kept in the final catalog. The extended $\mathrm{X}$-ray sources found in this way were visually screened and any obvious artifacts, often due to out-of-time (OOT) events or remaining background structure, were manually rejected. The optical images were visually inspected for possible counterparts of extended sources. In this step extended sources obviously associated with nearby galaxies were removed from the catalog. Grades were assigned to the quality of a cluster detection based on visual inspection of the optical and X-ray images. Cluster candidates with grade "+" are obvious real clusters, often the ones one would select by eye. Extended X-ray sources graded with "o" are possible clusters but not as prominent as those graded with "+". This grade is assigned to clusters without a very obvious optical counterpart but a reliable X-ray detection. In some cases these may be systems at very high redshift that are just barely visible in the optical images. Clusters graded "-_" appear to be unreliable in the optical and X-ray images, but were not rejected as obvious spurious sources based on their visual impression in the X-ray images.

The full X-ray cluster catalog is available in electronic format from the CDS. Cluster candidates in this catalog follow the naming scheme BLOX JHHMM.m+DDMM.m, where BLOX is the IAU registered acronym for "Bonn Lensing, Optical, X-ray" detected cluster candidates. In the following we give comments on individual fields when appropriate.

- RX J0505.3-2849 - Two extended X-ray peaks are each found on both previously known RX J clusters in this field. In the case of RX J0505.3-2849, we only list one; the other is probably a confusion with a double point source;

- RBS 0864 - Two reductions of this field were done. One reduction excluded the target cluster from the analysis, while the other one was made with the cluster remaining in the data. A large number of spurious detections associated with OOT events were manually rejected.

- MS 1054.4-0321 - Two extended X-ray sources were detected on the target cluster at about the same distance from the optical center of the cluster. We list both X-ray sources in the catalog.

- HE 1104-1805 - The only extended X-ray source found in this field coincides with a bright star.

- LBQS 1228+1116 - The X-ray data on this field is strongly affected by background flares. No clusters were found in the remaining shallow data.

- MKW 9- The target cluster dominates the center and several extended sources are detected in the cluster region, some of them on bright and large galaxies.

- QSO B1246-057 - The calibration problem that prevented the X-ray data on this field from being included in the public data release (Dietrich et al. 2006) were solved and the field could be included in the cluster search.

- A 1882 - The X-ray image of this cluster shows three very extended sources centered around the nominal position of the cluster of Abell (1958). These regions of extended X-ray emission were excluded from the analysis.

- NGC 7252 - No extended targets were found in this field. 


\section{Optical matched filter detection}

Clusters of galaxies can be optically selected in a multitude of ways, either from one passband alone or by combining color information from two or more passbands with the positional information on galaxies used in all methods. A review of a large number of optical detection methods has recently been published by Gal (2006).

For this work we chose the matched filter detection algorithm of Postman et al. (1996). The P96 method was selected because it is well-tested and efficient, it works on single passband catalogs, and can thus be used for the entire area of the XFS. More elaborate detection schemes using multi-color information will be employed on XFS data in subsequent work (Dietrich et al., in preparation).

\subsection{Optical catalog creation}

The starting point for any optical cluster-search method is a catalog of galaxies. It is thus only prudent to discuss the creation of such catalogs in some detail before turning to a short description of the matched filter algorithm and how it was implemented for this survey.

Unfortunately, WFI images are especially prone to internal reflections, producing prominent reflection rings around all saturated stars with blooming in the core. The brightest stars show more than one reflection ring, with increasing sizes and offsets from the position of the source. Additionally, extended halos and diffraction spikes occur around bright sources. The catalog creation tries to mask regions affected by these problems and do so as automatically as possible, but it still requires a large amount of manual masking.

The catalog production uses SExtractor (Bertin \& Arnouts 1996) and starts with a very low signal-to-noise ratio (SNR) catalog. The weight images produced by the GaBoDS pipeline are used in all steps of the catalog creation. The sole purpose of this first catalog is to identify regions that should be masked. Masked regions will be passed on to SExtractor in a FLAG image.

All objects of the initial catalog are put into cells of a grid whose size is chosen such that the average number of objects per cell is 2. This grid is smoothed with a Gaussian kernel with an FWHM of 1.4 pixels. We call the smoothed array $\mathcal{S}$. Automatically adjusting the grid size such that a fixed number of galaxies per cell is reached on average allows one to keep the size of the Gaussian, which is necessary to reach the desired SNR in the object density distribution, as fixed in the program. The main advantage of this approach is that the values of the kernel can be stored in a matrix (fixed to a $5 \times 5$ array in the program). The convolution of the density grid with the kernel matrix is computationally much faster than re-evaluating the Gaussian kernel at each grid cell. In the next step the dynamic range $\mathcal{S}$ is limited. Every pixel with a value $>1.5$ is set to 2 . Every pixel with a value $<1$ is set to zero; these pixels will be masked because they are either in very low SNR parts of the image, such as the edges, or they are covered by extended objects, such as large foreground galaxies or very bright stars. The resulting array is called $\mathcal{D}$.

Additionally, any rapid change of object density in $\mathcal{D}$, such as seen at the edges of reflection rings, is detected with a Sobel edge detection, i.e., an array containing the absolute values of the gradient of the array $\mathcal{D}$. The gradient computation is implemented as a convolution of $\mathcal{D}$ with the two $3 \times 3$ convolution kernels that correspond to finite second-order, two-sided differentiation. Every pixel exceeding a threshold in $\mathcal{D}$ is flagged in the output image.

Finally, the FLAG array, containing only values of 1 for pixels to be flagged and 0 for all other pixels, is smoothed again with the 1.4-pixel FWHM Gaussian to account for the fact that the initial smoothing shrinks the areas not covered by objects. Every pixel with a non-zero flag value will be flagged in the output FLAG image, which is expanded to the size of the original WFI image from which the catalog was created.

FLAG images created through this procedure reliably mask extended objects, bright stars, and the most prominent reflection rings. Exceptionally empty regions on the sky are only rarely masked erroneously. However, fainter reflection rings and stars of intermediate magnitude must still be masked by hand. Files describing the regions masked manually - either by circles or polygons for more complex shapes - must be generated by the user with tools such as the image viewer DS9. From them and the automatically generated FLAG image, the final FLAG image used in further steps in the catalog production is created.

The image seeing is determined from a high-SNR catalog. A histogram of the $F W H M$ of all objects with $0 . ' 3<F W H M<$ 3 .' 0 is created and the image seeing is set equal to the FWHM of the bin with the most objects. The seeing is used as input for SExtractor's star-galaxy classifier CLASS_STAR. Since we are only interested in galaxies and not in stars, reliably separating them in the science-grade catalogs is important for galaxy cluster searches.

Finally, two science-grade catalogs are created with SExtractor. Their only difference is the filter with which the detection image is convolved. The catalog for the optical matched filter search is made using a Gaussian kernel with an $F W H M$ of 4 pixels. This kernel ensures that relatively few spurious detections of faint objects are made, but it has a lower completeness at the low SNR end with a tendency to miss very small objects. Another catalog with SExtractor's default filter - a $3 \times 3$ pixel pyramidal kernel - is created as the starting point for the weak-lensing catalog creation (see Sect. 5).

\subsection{The Postman matched filter catalog}

The Postman matched filter algorithm is described in detail in P96. The main features of this algorithm separating it from other single-band cluster detection schemes are (1) it uses photometric and not only positional information; (2) the contrast of structures that approximate the filter shape with the background is maximized; (3) redshift and richness estimates of cluster candidates are produced as a byproduct; (4) the algorithm performs well over a wide range of redshifts. The main disadvantage is that a particular radial profile and luminosity function must be assumed. Clusters deviating from the expected profile will be detected only at lower significances or suppressed. Our implementation follows the description of P96 and Olsen et al. (1999, O99 hereinafter) with some modifications of the selection parameters as outlined below.

A series of programs and shell scripts is used to go from the object catalogs described in Sect. 4.1 to a catalog of cluster candidates. The input catalog is filtered such that only objects with a high probability of being galaxies are kept. To this end only objects with a CLASS_STAR value $<0.5$ are kept. At the faint end the catalog is cut at a magnitude $10 \sigma$ above the sky background as measured in a $2^{\prime \prime}$ aperture to ensure a high completeness of the catalog. Objects brighter than 17 mag are also filtered. These are often bright stars, which are not correctly identified as such by SExtractor and would cause a serious contamination of the 
input catalog. If any of these objects with $R<17$ are bright, nearby cluster members, the matched filter signal of the cluster is decreased. However, the fainter cluster galaxies usually still lead to a significant detection of the galaxy cluster but with a redshift bias. This bias is introduced because omitting the bright cluster galaxies modifies the luminosity function. This redshift bias introduced by the cut on the bright galaxies is marginal, at least for the redshift range considered here.

Likelihood maps $S(x, y)$ are computed on a grid and saved as FITS images for a series of redshifts. We compute $S$ with a spacing in redshift of $\Delta z=0.1$, starting at $z=0.1$ and up to the last output grid in which the $m^{*}$ of the fiducial cluster model shifted to that redshift is not fainter than the limiting magnitude of the input catalog. For the XFS R-band observations, this is typically up to $z=0.9$. Following the prescription of O99, we assume a fiducial cluster model at $z=0.6$ with a Schechter luminosity function with $M_{R}^{*}=-21.63 \mathrm{mag}$, faint end slope $\alpha=-1.1$, and a Hubble profile with physical core radius $R_{\mathrm{c}}=0.1 h_{70} \mathrm{Mpc}$ integrated out to the cutoff radius $r_{\infty}=10 R_{\mathrm{c}}$. We keep the pixel size of the output grid constant at $0.5 \mathrm{pix} / R_{\mathrm{c}}$ at $z=0.6$ but vary the area required for a detection in the output images with redshift. The $k$-correction needed for the redshift dependence of $m^{*}$ is computed for an elliptical galaxy with no evolution.

Having created the likelihood maps, we are now faced with the problem of identifying clusters of galaxies in them; i.e., we have to decide which peaks in the likelihood maps are reliable candidates for galaxy clusters. Peaks are detected with SExtractor. The main SExtractor detection parameters were adopted from $\mathrm{O} 99$ and are set as follows:

- the minimum area for a detection MIN_AREA scales with the redshift and corresponds to $\pi r_{\mathrm{c}}^{2}$, where $r_{\mathrm{c}}$ is the size of core radius $R_{\mathrm{c}}$ projected on the sky;

- the detection threshold DETECT_THRESH is set to 2;

- no deblending of peaks is performed (DEBLEND_MINCONT = 1), so that all contiguous pixels above the detection threshold are counted as one cluster candidate;

- a global background is estimated from the image.

Catalogs from the individual output grids at different redshifts are then matched by position using the LDAC program associate. Peaks present in at least 3 output grids with a minimum significance of at least $3.5 \sigma$ in one of them are kept as reliable cluster candidates. These parameters are slightly different from the ones adopted by O99, who used a threshold of $3 \sigma$, a detection in at least 4 output grids, and a minimum value of the richness parameter $\Lambda_{\mathrm{cl}}$. The reason for different selection criteria are the different redshift regions of interest in the study of 099 and the work presented here. While O99 are chiefly interested in high redshift clusters, our search mainly aims for intermediate redshift clusters accessible with weak lensing, i.e., most of these clusters will be at redshifts $z=0.2 \ldots 0.3$. Several of the criteria adopted by 099 are biased against the search for clusters at intermediate redshifts.

The significance of a cluster signal drops sharply once we look at output grids at redshifts higher than the cluster's redshift because the power law cutoff of the luminosity filter strongly suppresses signals from lower redshifts. For clusters at lower redshifts, the number of output grids at redshifts lower or equal to the cluster redshift is small. The requirement of O99 that a cluster candidate be detected in at least 4 output grids is thus heavily biased against the detection of clusters at the redshift interval we are especially interested in. We therefore relaxe the requirement on the number of output grids in which a cluster must be detected to 3 , and have the lowest redshift output grid at $z=0.1$ instead of $z=0.2$. To compensate for the higher number of spurious detections caused by this less stringent cut, the minimum significance to be reached in at least one output grid is increased from 3 to 3.5 .

We do not make any cuts on the richness parameter $\Lambda_{\mathrm{cl}}$. Clusters at higher redshifts need to be much more luminous than clusters at lower redshifts to be detectable. Consequently, the average richness parameter of clusters at higher redshift is much larger than that of lower redshift clusters. Imposing a minimum value for $\Lambda_{\mathrm{cl}}$ would create a strong bias against low and intermediate redshift systems.

The selection criteria adopted by $\mathrm{O} 99$ have proven to be very successful by a high rate of spectroscopically confirmed clusters found in the EIS wide survey (e.g., Hansen et al. 2002; Olsen et al. 2003; Benoist et al. 2002) and we are thus confident that our slightly modified criteria are also successful. However, we have to note that the simulations on which these criteria were developed are not able to predict the number of spurious detections. O99 attempted to simulate a pure background population of galaxies by randomizing the position of galaxies while keeping their magnitude fixed. This randomized population has a much smoother distribution than the field population of galaxies. The reason is that the distribution of field galaxies is not a random field with white noise, but instead shows clustering and correlation.

More recently, Olsen et al. (2007, O07) have used a more realistic approach by creating backgrounds that are correlated within bins of 1 mag following the prescription of Soneira \& Peebles (1978). Their selection parameters are a minimum significance of $3.5 \sigma$, minimum area of $\pi r_{\mathrm{c}}^{2}$, and number of redshift slices $>1$, which are very similar to ours.

We applied the matched filter algorithm to R-band catalogs from 23 fields of the XFS. The resulting catalog is available in electronic format from the CDS. It lists 116 candidate clusters. Thirteen of the systems in this catalog have been previously found by other authors and have either spectroscopic or photometric redshift information. All cluster candidates were visually inspected and graded as for to the grades given to the X-ray detected clusters. Cluster candidates with grade "+" are obvious real clusters, often the ones one would select by eye, or they have and extended X-ray source as a counterpart. Matched-filter peaks graded "o" are probable detections but less prominent than those graded "+" and may be more prone to projection effects. Candidates with a "-" grade are most likely artifacts or maybe in some cases very poor clusters or groups.

Out of the total of 116 matched-filter peaks 49, were graded “+”, 42 “०”, and 25 received a grade of "-_". There is a clear correlation between the significance of a cluster candidate and its grade. The average SNR of a "+" rated cluster is $5.5 \sigma$ and include the highest SNR detections; "o" graded cluster candidates have an average significance of 4.9 , while the unlikely candidates rated with "-" have an average value of $\bar{\sigma}_{\max }=4.4$.

In the field RBS 0864, two clusters were detected independently on both pointings on this field. These are BLOX J1023.6+ 0411.1 (RBS 0864 itself) and BLOX J1022.9+0411.9. Their properties reported in the table of matched-filter detected clusters at the CDS are averages of both independent detections.

A comparison of the matched-filter estimated redshifts of the 13 previously found systems with their redshift values found in the literature is provided by Fig. 1. Seven of these 13 clusters have spectroscopic redshift information, while the remaining 6 cluster have only photometric redshift estimates. We point out that the redshifts of the photometric sample are not photometric redshifts in the classical sense of, e.g., template fitting but 


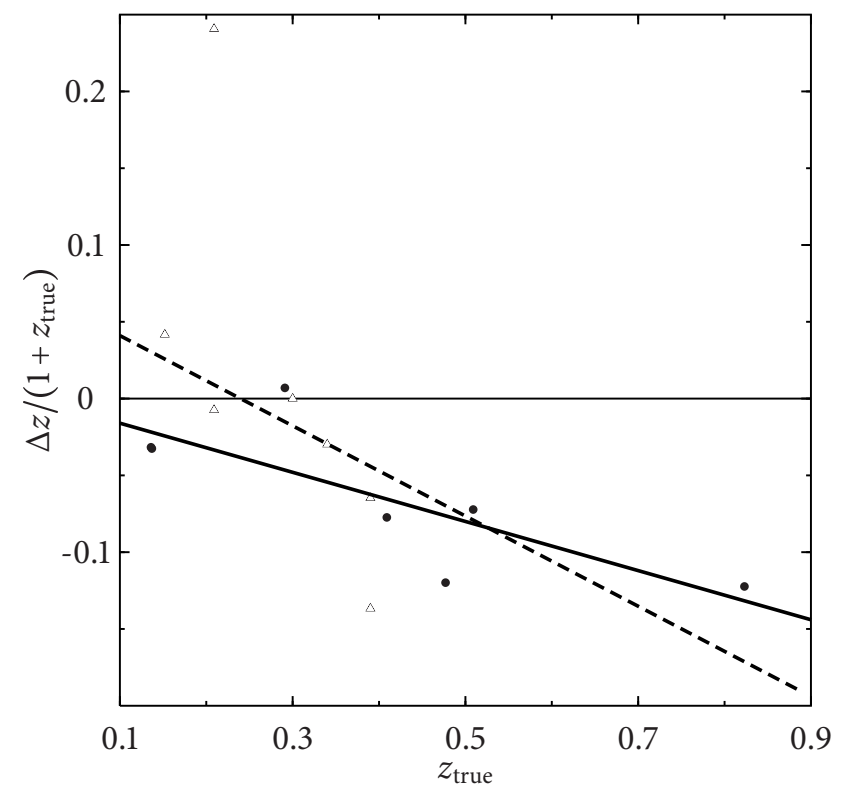

Fig. 1. Comparison of redshift estimates obtained from the P96 matched filter with literature values. Solid circles denote data points with spectroscopic redshift information; open triangles are photometric redshift estimates from the literature. The matched filter underestimates the redshift as is shown by the best-fit lines to the spectroscopic sample (solid line) and the full sample (dashed line).

are the redshift estimators of other cluster finding methods. This is most notably the redshift estimator of the "cut-and-enhance method" of Goto et al. (2002), which provides 4 redshifts in this sample. The mean offset from the zero line for the spectroscopic sample is marginally significant with $\left\langle\Delta z /\left(1+z_{\text {true }}\right)\right\rangle=-0.06 \pm$ 0.05 , while the mean offset of the whole sample $\left\langle\Delta z /\left(1+z_{\text {true }}\right)\right\rangle=$ $-0.06 \pm 0.07$ is consistent with zero, where the error is simply the standard deviation of the scatter. The relatively small deviation from zero hides a significant bias of the matched filter redshifts towards lower redshifts. The solid line in Fig. 1 is the best linear fit to the spectroscopic data points. The line is described by $\Delta z /\left(1+z_{\text {true }}\right)=(0.00 \pm 0.03)+(-0.16 \pm 0.06) z_{\text {true }}$. Thus, the deviation from the ideal relation is significant at the $2.7 \sigma$ level. The bias increases marginally if the photometric data points are included in the analysis. The best-fit line is then described by $\Delta z /\left(1+z_{\text {true }}\right)=(0.07 \pm 0.05)+(-0.29 \pm 0.12) z_{\text {true }}$.

Since the clusters were found on different fields with independent photometric calibration, errors in the photometric calibration can be excluded as the source of this systematic difference. Possible sources of the underestimated redshifts are (1) the $k$-correction, which depends on the adopted galaxy model and (2) the luminosity function of the fiducial cluster model, specifically the value of $M^{*}$. The discrepancy increases with higher redshifts. Our flux filter was constructed under the assumption of no evolution and thus stronger discrepancies are indeed expected at higher redshifts. This has already been noted by P96, who also found the estimated redshifts of higher redshift clusters to be systematically too low by about $\Delta z=0.1 \ldots 0.2$.

The 007 paper carried out a matched-filter cluster search in the Canada-France-Hawaii Telescope Legacy Survey (CFHTLS) Deep. The CFHTLS field D4 coincides with our field LBQS2212-1759. We briefly compare the results of these two independent cluster searches. They find 6 matched-filter selected cluster candidates inside the WFI FOV. Two of their detections, CFHTLS-CL-J221500-175028 and

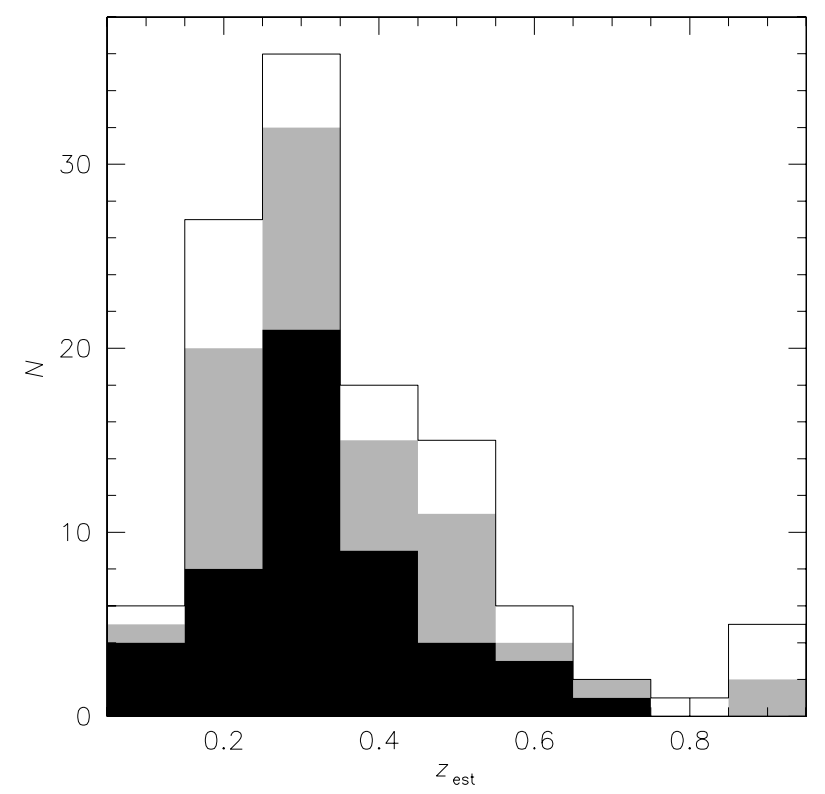

Fig. 2. Histogram of the estimated redshift distribution of the matchedfilter detected clusters. The open histogram represents all cluster candidates, the gray histogram the "+" and "o" rated candidates, and the black histogram only the "+" rated clusters.

CFHTLS-CL-J221537-174533, coincide with matchedfilter cluster candidates, BLOX J2215.0-1750.5 and BLOX J2215.6-1745.5, detected in this survey. Both cluster candidates received the best grade in either survey. The matched filter redshift estimates agree for CFHTLS-CLJ221500-175028/BLOX J2215.0-1750.5, while the O07 put CFHTLS-CL-J221537-174533/BLOX J2215.6-1745.5 slightly higher at $z=0.4$ than our estimate of $z=0.3$. Two other cluster candidates of $\mathrm{O} 07$ coincide with possible clusters we found with other methods. CFHTLS-CL-J221620-173224, an "A" rated potential cluster at an estimated redshift of $z=0.7$ matches our weak lensing detection (see Sect. 5) BLOX J2216.3-1733.0. CFHTLS-CL-J221606-175132, a "B" rated cluster candidate with a redshift estimate of $z=0.4$ matches our weak lensing detection BLOX J2216.1-1751.7. Our matched-filter cluster candidate BLOX J2214.4-1728.1 at $z=0.2$ is not found by $\mathrm{O} 07$.

\section{Weak lensing detection}

The tidal gravitational field of a galaxy cluster causes a coherent tangential of the sheared images of background galaxies. A quantitative measure for this alignment, the aperture mass $M_{\text {ap }}$, was developed in Schneider (1996). There, $M_{\text {ap }}$ is defined as a weighted integral over the dimensionless surface mass density $\kappa$

$M_{\mathrm{ap}}\left(\boldsymbol{\theta}_{0}\right)=\int_{\sup U} \mathrm{~d}^{2} \theta U(\vartheta) \kappa(\boldsymbol{\theta})$,

where $U(\vartheta)=U\left(\left|\boldsymbol{\theta}-\boldsymbol{\theta}_{\mathbf{0}}\right|\right)$ is a radially symmetric weight function with zero total weight to avoid the mass-sheet degeneracy (Schneider \& Seitz 1995). The aperture mass in Eq. (1) is defined in terms of the surface mass density, but it is possible to find an expression that allows computing $M_{\text {ap }}$ in terms of the observable shear $\gamma$ :

$M_{\mathrm{ap}}\left(\boldsymbol{\theta}_{0}\right)=\int_{\sup Q} \mathrm{~d}^{2} \theta Q(\vartheta) \gamma_{\mathrm{t}}\left(\boldsymbol{\theta} ; \boldsymbol{\theta}_{0}\right)$ 
where we define the tangential shear $\gamma_{\mathrm{t}}$ relative to a point $\boldsymbol{\theta}_{0}$ by

$\gamma_{\mathrm{t}}\left(\boldsymbol{\theta} ; \boldsymbol{\theta}_{0}\right)=-\mathfrak{R}\left[\gamma\left(\boldsymbol{\theta}+\boldsymbol{\theta}_{0}\right) \mathrm{e}^{-2 \mathrm{i} \varphi}\right]$.

Here, $(\vartheta, \varphi)$ are polar coordinates with respect to $\boldsymbol{\theta}_{0}$, and the weight function $Q(\vartheta)$ is related to $U(\vartheta)$ by

$Q(\vartheta)=\frac{2}{\vartheta^{2}} \int_{0}^{\vartheta} \mathrm{d} \vartheta^{\prime} \vartheta^{\prime} U\left(\vartheta^{\prime}\right)-U(\vartheta)$.

On real data the aperture mass can be estimated by a sum over the $N_{\mathrm{g}}$ galaxy ellipticities inside the aperture,

$M_{\mathrm{ap}}\left(\boldsymbol{\theta}_{0}\right)=\frac{1}{n} \sum_{i=1}^{N_{\mathrm{g}}} Q\left(\vartheta_{i}\right) \varepsilon_{\mathrm{ti}}$.

Here $n$ is the number density of faint background galaxies (FBG), and the tangential component of the ellipticity has been defined in analogy to Eq. (3).

The SNR of a peak in maps of aperture mass can be estimated by using the fact that $\left\langle M_{\mathrm{ap}}\right\rangle \equiv 0$ holds in the case of no lensing. Then the rms dispersion of the $M_{\mathrm{ap}}$-statistic becomes $\sigma_{M_{\text {ap }}}=\sqrt{\left\langle M_{\text {ap }}\right\rangle^{2}}$, which is

$\sigma_{M_{\mathrm{ap}}}=\frac{\sigma_{\varepsilon}}{\sqrt{2} n}\left[\sum_{i=1}^{N_{\mathrm{g}}} Q^{2}\left(\theta_{i}\right)\right]^{1 / 2}$,

using

$\left\langle\varepsilon_{i} \varepsilon_{j}\right\rangle=\frac{\sigma_{\varepsilon}^{2}}{2} \delta_{i j}$

What remains to be fixed is the shape of the weight function. If only white noise caused by the random ellipticity of background galaxies is present, i.e., if the noise is described by Eq. (6), the filter function $Q$ should follow the shear profile of the cluster as closely as possible to increase the SNR of a galaxy cluster detection. The aperture mass then becomes a matched filter technique for weak lensing detections of galaxy clusters.

$\mathrm{N}$-body simulations predict the shape of collapsed halos to follow an NFW profile. The shear of an NFW profile can be computed analytically (Bartelmann 1996; Wright \& Brainerd 2000), but the resulting expressions are complex and time-consuming to evaluate. Schirmer et al. (2007) have proposed a simple approximation to the NFW shear profile,

$Q_{\mathrm{NFW}}(x)=\frac{\tanh x}{x}$

that is much faster to compute than the full expression. The foregoing equation is not used directly for the computation of $M_{\text {ap }}$, but exponential cut-offs are multiplied to Eq. (8) as $x \rightarrow 0$ and $x \rightarrow \infty$,

$Q_{\mathrm{NFW}}\left(x ; x_{\mathrm{c}}\right)=\frac{1}{1+\mathrm{e}^{6-150 x}+\mathrm{e}^{-47+50 x}} \frac{\tanh \left(x / x_{\mathrm{c}}\right)}{x / x_{\mathrm{c}}}$.

The purpose of these cut-offs is (1) to avoid finite field effects as the weight function (8) formally extends to infinity, but data is only available on a finite field; and (2) to downweight the signal close to the cluster core where cluster dwarf galaxies may pollute the signal and where the reduced shear $g$ rather than the shear $\gamma$ should be used. The parameter $x_{\mathrm{c}}$ in Eq. (8) controls the shape of the filter function. For low values of $x_{\mathrm{c}}$, more weight is put to smaller filter scales. In the absence of the exponential cut-offs, variations in $x_{\mathrm{c}}$ and $\theta_{\max }$ are obviously degenerate, but the exponential cut-off makes the weight function (9) a two-parameter family. Hetterscheidt et al. (2005) find that the optimal value of $x_{\mathrm{c}}=0.15$ for a range of cluster masses and redshifts, so we will use this value throughout this work.

The projection of large-scale structure (LSS) along the line of sight can potentially be a serious contaminant for every weak lensing observation of galaxy clusters. Such projections of sheets and filaments inevitably lead to false cluster detections at all significance levels expected from real clusters (HS05) and missed cluster detection except for the most massive systems (H04). It was shown by HS05 that, even in the absence of shape noise, the efficiency of a weak lensing search for galaxy clusters does not exceed $\sim 85 \%$ and also depends on the shape of the filter function.

Maturi et al. (2005) propose to treat the projections of masses in the background and foreground of the cluster, i.e., the largescale structure as a source of non-white noise. They constructe an optimal filter that tries to maximize the signal caused by a galaxy cluster while downweighting the cosmic shear signal on scales of interest. The resulting filter function $Q_{\mathrm{LSS}}$ depends not only on the expected shear profile of the galaxy cluster but also on the number density of FBG and on the convergence power spectrum to describe the expected noise. The latter noise contribution in turn depends on the redshift distribution of the FBG. In the case of pure white noise, the Maturi filter takes the shape of an NFW shear profile, i.e., essentially the same form as the Schirmer filter, and both filters are virtually equivalent. If projections of the LSS are a significant noise source, then using $Q_{\mathrm{LSS}}$ instead of $Q_{\mathrm{NFW}}$ can lead to significantly lower contaminations of the cluster catalog with false positives.

\subsection{Signal and noise of the aperture mass}

Before we apply the aperture mass statistic to the XFS data we need to understand the properties of the aperture mass function in some more detail. Equations (5) and (6) allow us to compute the SNR of $M_{\text {ap }}$ as

$S=\frac{\sqrt{2} \sum_{i} Q_{i} \varepsilon_{\mathrm{t} i}}{\sqrt{\sum_{i} Q_{i}^{2} \varepsilon_{i}^{2}}}$

where $Q_{i}$ is the weight assigned to the $i$ th galaxies by the radially symmetric weight function $Q(\vartheta)$. This expression, however, considers only the noise caused by the random ellipticities and not the shot noise of the finite sampling of the $M_{\text {ap }}$ statistic by the population of background galaxies.

We used ray-tracing simulations to examine these effects in detail. These were generated by tracing light rays through the $\Lambda$ CDM $N$-body simulation of the VIRGO consortium (Jenkins et al. 1998). These $N$-body simulations have been carried out with the following parameters: $\Omega_{\mathrm{m}}=0.3, \Omega_{\Lambda}=0.7, h_{70}=1$, $\sigma_{8}=0.9, \Gamma=0.21$, and the index of the primordial power spectrum $n_{\mathrm{s}}=1$. The population of background galaxies has been assumed to be a $\delta$-function peaked at $z=1$.

We now give a short description of our ray-tracing algorithm. More details of can be found in Hartlap (2005). From redshift 0 to the source redshift, $1024^{2}$ light rays are traced through 16 slices of $202.9 h_{70}^{-1} \mathrm{Mpc}$ thickness onto an output grid of $1 \times$ 1 square degree. Each redshift slice corresponds to one output box of the $N$-body simulation and is projected as a whole onto a lens plane, preserving the periodic boundary conditions of the $\mathrm{N}$-body box. To avoid repetition of structure along the line of 
sight, the planes are randomly shifted and rotated. The light rays are shot from the observer through the set of lens planes, forming a regular grid on the first plane. We then use FFT methods to compute the lensing potential on each lens plane, from which we obtain the deflection angle and its partial derivatives on a grid. The ray position and the Jacobian of the lens mapping for each ray are obtained by recursion. Given the ray position on the current lens plane, its propagation direction (known from the position on the last plane), and the deflection by the current plane interpolated onto the ray, we immediately obtain the ray position on the next plane. Differentiation of this recursion formula with respect to the image plane coordinates yields a similar relation for the Jacobian of the lens mapping, taking the tidal deflection field computed before into account. The recursion is performed until we reach the source plane. From the final Jacobian, we obtain noiseless convergence and shear maps in the usual way.

Since we wish to study which convergence $/ M_{\text {ap }}$ peaks correspond to real dark matter halos, we also have to compute the lensed positions of the central particles of the dark matter halos contained in the halo catalogs. We achieve this by projecting the halo position onto the lens planes and identifying the light ray that passes closest to the halo. The lensed position of the halo is then computed by inverting the linearized lens mapping around this ray.

Fifty different realizations were made by using in each case different random shifts and rotations of the lens planes. Lensing catalogs were created by randomly distributing galaxies with an ellipticity dispersion of $\sigma_{\varepsilon}=0.38$ over the output grid of the ray-tracing simulations on areas corresponding to the sizes of actual XFS fields until the number density of the respective XFS field was reached. When placing galaxies, the masks used in the catalog generation of the real data were applied to simulated catalogs as well, to realistically model the effect of holes in the field. Three different masks and number densities were taken from the XFS data. All masks were put on each of the 50 raytracing realization to obtain a high number of lensing simulations. The effective (unmasked) area and number densities of these fields are $970 \operatorname{arcmin}^{2}$ and $13.4 \operatorname{arcmin}^{-2}, 1022 \operatorname{arcmin}^{2}$ and $17.7 \mathrm{arcmin}^{-2}$, and $981 \mathrm{arcmin}^{2}$ and $18.2 \mathrm{arcmin}^{-2}$. The total area covered by our ray-tracing simulations is $41.3 \mathrm{sq}$. deg.

With typical sizes of the XFS fields of $35^{\prime} \times 35^{\prime}$, the individual lensing simulations are not totally independent but have some overlap because the side length of one ray-tracing simulation is only $60^{\prime}$, not enough to accommodate two XFS masks next to each other. The catalogs were placed on the ray-tracing simulations such that this overlap is minimized. While not completely independent, these overlapping areas were covered by catalogs with different maskings, different number densities, and different realizations of Gaussian noise so that for our purpose understanding the noise properties of $M_{\text {ap }}$ from realistic simulations - no significant correlation between individual lensing simulations is expected.

We computed the aperture mass for 9 different filter scales and the filter functions proposed by Schirmer et al. (2007) and Maturi et al. (2005) from the same catalogs. As both filter functions are based on an NFW model of a cluster, we chose the filter radii based on an assumed fiducial cluster model. In this model the cluster is at redshift $z=0.3$, the redshift at which we expect the lens strength in our survey to be maximized. Table 2 gives these filter radii and the corresponding virial mass if the filter scale is interpreted as the virial radius of the cluster. We also need to fix the redshift of the source galaxies to model the large-scale structure power spectrum in the Maturi filter. We assumed that all background galaxies are at $z_{\mathrm{s}}=0.8$ and that the
Table 2. Filter radii for $M_{\text {ap }}$ computation and corresponding virial mass.

\begin{tabular}{rl}
\hline \hline $\begin{array}{r}\text { Radius } \\
\left(\mathrm{kpc} / h_{70}\right)\end{array}$ & $\begin{array}{c}M_{\text {vir }} \\
\left(10^{14} h_{70}^{-1} M_{\odot}\right)\end{array}$ \\
\hline 1000 & 0.76 \\
1247 & 1.5 \\
1493 & 2.5 \\
1740 & 4.0 \\
1986 & 6.0 \\
2233 & 8.5 \\
2479 & 12.0 \\
2726 & 15.0 \\
2972 & 20.0 \\
\hline
\end{tabular}

number density of background galaxies is $n=18 \operatorname{arcmin}^{-2}$. We computed $M_{\text {ap }}$ on a grid and set the pixel size in this grid such that one pixel corresponds to $50 h_{70}^{-1} \mathrm{kpc}$ at the redshift of the fiducial cluster model.

For all lensing simulations and filter scales we computed $M_{\text {ap }}$ and $-M_{\text {ap }}$ (for later detection of negative peaks as a control) for both filter functions from the input catalog and $M_{\text {ap }}$ after rotating (1) all galaxies by $45^{\circ}$; (2) every galaxy by a random angle for the Schirmer filter. Maps of the aperture mass and their SNR ( $S$-maps), which were computed as well, were saved as FITS images.

As for the detection of peaks in the matched filter maps, we used SExtractor to identify shear-selected peaks in the $M_{\text {ap }}$-maps. For this purpose we run SExtractor in dual-image mode with the $M_{\text {ap }}$-map as the detection image and the $S$-map as the measurement image. This means that the SNR of a $M_{\text {ap }}$ peak is computed with Eq. (10) and not determined by SExtractor. This is a small difference from the matched filter pipeline, in which we did not use the P96 likelihood to determine a significance but used SExtractor detection significances.

The detection threshold is set to $2 \sigma$ and the minimum detection area scales with the filter such that it corresponds to the pixels covered by a circle with a radius twice as large as the filter scale. SExtractor is run without deblending, i.e., every contiguous area above the detection threshold is counted as one object. Peaks found in this way in different filter radii were associated based on positional coincidence.

We first examine the results obtained with the Schirmer filter before comparing these to the ones obtained with the Maturi filter. Positional offsets between the weak lensing peak positions and the position of the BCG or the center of X-ray emission from the hot intracluster gas are commonly observed (Wittman et al. 2006) and expected (Dietrich et al. 2005). The size of the offsets has been studied for the case of an isolated SIS by Dietrich et al. (2005). The ray-tracing simulations used here allow us to investigate the additional effect of large-scale structure along the line-of-sight.

The catalogs of weak lensing halos produced in this way from the $M_{\text {ap }}$ images were associated with a catalog of actual dark matter halos in the VIRGO simulation. For this purpose we only considered halos with masses in excess of $10^{14} M_{\odot}$ and with redshifts $0.1<z<0.7$ as these are roughly the ones to which we expect to be sensitive in our galaxy cluster survey. The maximum distance allowed for a match between halo position and $M_{\text {ap }}$ peak was determined by the virial radius of the halo and the size of the $M_{\text {ap }}$ peak as determined by SExtractor. Note that the position of the halo was derived from the most-bound particle in that halo identified by the friend-of-friend halo finder employed to generate the halo catalog. In rare cases this is in the 


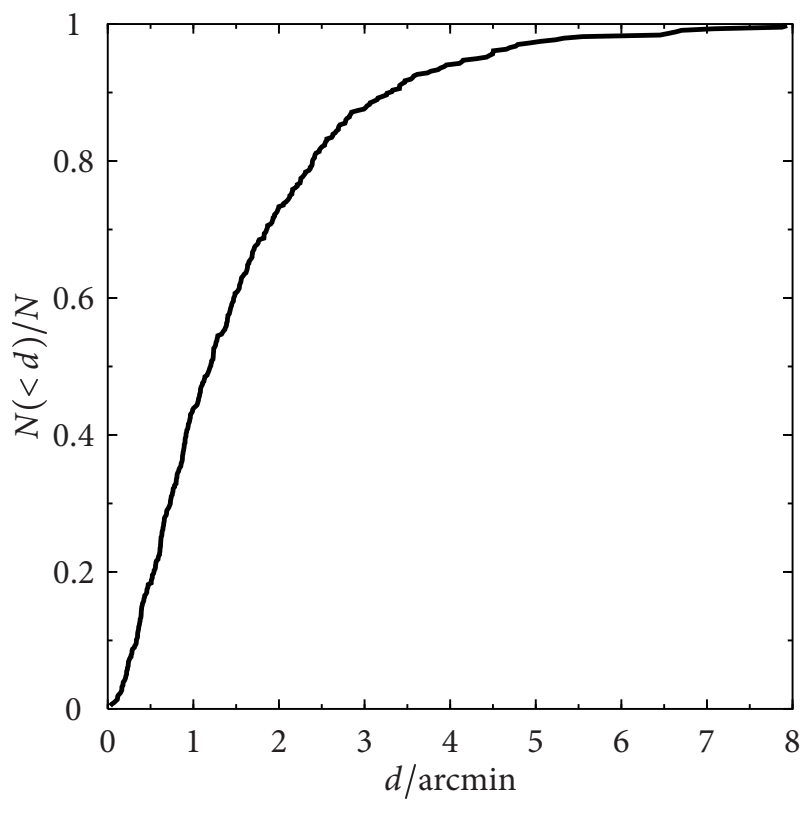

Fig. 3. Cumulative distance distribution of the $434 M_{\text {ap }}$ peaks that could be associated to a dark matter halo in the VIRGO simulation. $75 \%$ of all matches are made within a 2'.15 radius.

center between what by eye be would identified as two separate halos.

In the ray-tracing simulations, 434 peaks in the $M_{\mathrm{ap}}$-maps could be associated with dark matter halos in the VIRGO simulation. Figure 3 shows the cumulative distribution of their positional differences. From the number density of $M_{\text {ap }}$ peaks and the average size of the association radius we estimate that $\sim 100$ or $\sim 25 \%$ of those matches are chance coincidences. Inspection of Fig. 3 shows that $75 \%$ of all positional offsets are smaller than 2 '.15, which is the maximum offset we will allow from here on. Note that on the one hand this is significantly smaller than the 3 ' matching radius adopted by HS05, who used a higher number density and a smaller ellipticity dispersion. On the other hand, it is significantly larger than the offsets found for an isolated SIS by Dietrich et al. (2005). This possibly indicates a non-negligible influence of large-scale structure along the line of sight on the weak lensing peak positions of dark matter halos. One, however, has to be careful when drawing this conclusion as we are looking at halos at very different redshifts, while Dietrich et al. (2005) studied only systems at one redshift.

Aperture mass peaks not associated with dark matter halos can be caused either by projections of large-scale structure mimicking a shear signal of a cluster or by the shape noise of background galaxies that can cause random tangential alignments. In real data the measured ellipticities must be corrected for atmospheric seeing and PSF distortions of the instrument. Residuals in this correction can lead to spurious alignments of background galaxies, both in curl-free shear fields (pure E-modes) and in non-curl free shear fields (also including $B$-modes). E-modes are transformed into B-modes (and vice versa) by rotating all galaxies by $45^{\circ}$. Because gravitational lensing only creates E-modes, the observed power of B-modes is often used as a quality check of the PSF correction. We did not check our lensing signal for the presence of B-modes. However, cosmic shear studies using PSF correction schemes very similar to ours find that B-modes typically occur on scales smaller than are relevant for our $M_{\text {ap }}$ kernels (e.g., Van Waerbeke et al. 2005; Hetterscheidt et al. 2006).

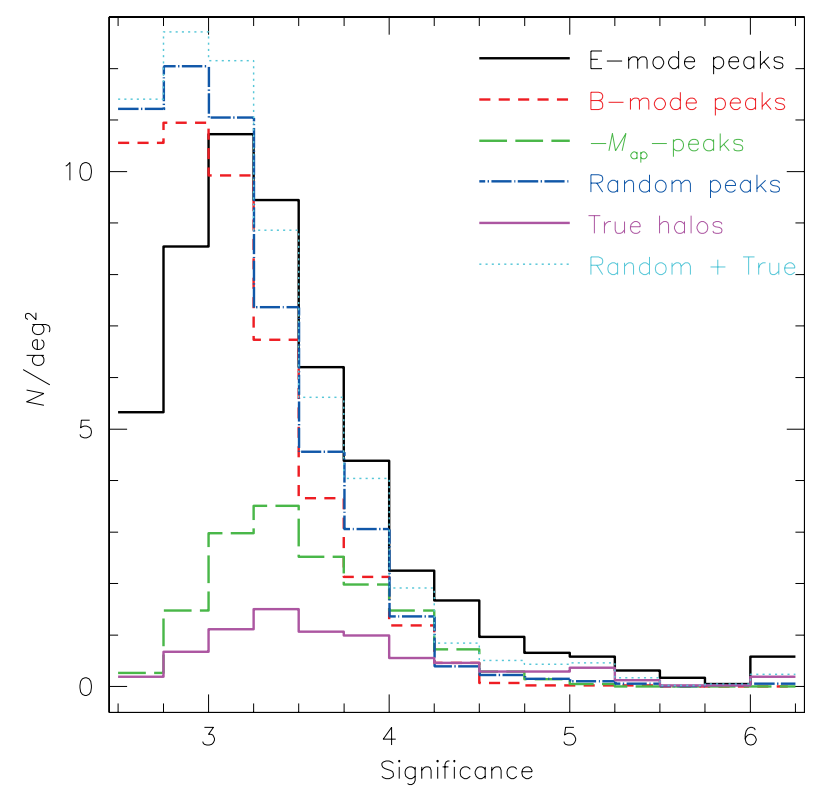

Fig. 4. Peak significances of shear selected peaks in the simulated maps of $M_{\text {ap }}$ (black solid), $-M_{\text {ap }}$ (green long dashed), B-mode $M_{\text {ap }}$ (red short dashed), and mock catalogs (blue dot-dashed). The solid pink line corresponds to peaks successfully associated with dark matter halos within 2 ' 15 . The thin light-blue dotted line is the sum of the random peaks (blue) and the true halos (pink).

It is thus safe to assume that we can apply the results of our ray-tracing simulations to the XFS.

Figure 4 shows the significance distribution of shear-selected peaks in the different kinds of aperture mass maps created with the Schirmer filter. These are (1) the distribution of peak significances for all $M_{\text {ap }}$ peaks created from the ray-traced catalog, i.e., those peaks one would find in real data; (2) negative peaks or, considering how the peak finding pipeline is run, peaks found in $-M_{\text {ap }}$-maps; (3) peaks found in B-mode $M_{\text {ap }}$-maps, i.e., maps of aperture mass created after all galaxies in the ray-traced catalog were rotated by $45^{\circ}$; (4) weak lensing peaks that could be associated with dark matter halos within a matching radius of 2.15 ; and (5) peaks found in mock catalogs with random ellipticities. It is important to emphasize that the random seed was kept fixed so that when computing $M_{\text {ap }}$ on different filter scales the orientation of galaxies in the input catalog remained unchanged. Here we consider only those peaks that were detected on at least two filter scales. We justify this choice later when examining the influence of the number of filter scales in which peaks are detected. The significance used in the histogram is the maximal significance values of all filter scales a peak was detected in.

A number of features in Fig. 4 are worth a more detailed discussion. First, we note that the number density of peaks in the $M_{\text {ap }}$ - and $-M_{\text {ap }}$-maps declines towards lower significances, while in the B-mode and random maps it remains roughly constant below $3.25 \sigma$. Naively, one would expect an increase in all curves towards lower significances. The observed behavior is due to a selection bias when running SExtractor on the FITS images. The detection threshold is derived from the standard deviation of the background in these images. The B-mode and random maps are overall flatter than the E-mode $M_{\text {ap }}$-maps. This leads to a detection threshold at which peaks of lower significance in the $\mathrm{S}$-maps are detected in the $M_{\mathrm{ap}}$-maps for random maps and B-mode maps than for E-mode maps.

Second, the number densities of random and B-mode peaks are very similar, with the latter slightly lower. As we did not 
simulate the systematic influence of instrumental PSF corrections, the only sources of B-modes are the shape noise of background galaxies and finite field effects in the $M_{\text {ap }}$ estimator. The effect of shape noise alone is simulated by the random catalogs, while the B-mode peaks are a combination of shape noise and systematic effects due to the finiteness of the field and holes in the data. The fact that the number density of B-mode peaks is compatible with the number density of random peaks shows that systematic effects contributing to B-modes are not an important noise source. This is confirmed by a visual inspection of the peak distribution indicating that B-mode peaks do not show an obvious tendency to appear close to holes in or edges of the data field.

Third, the number of E-mode peaks is higher than the number of any other peak statistic in all significance bins $>3.25 \sigma$ in which the aforementioned selection plays no role. The sum of the true halos peaks and the random peaks is compatible with the number of E-mode peaks in the significance bins from $3.25 \sigma$ to $4.25 \sigma$, if one assumes Poissonian statistics. At higher significances, an excess of E-mode peaks is observed. We surmise that this is due to projections of large-scale structures. This means that we can expect a significant fraction of spurious peaks at almost all significances, a result that is compatible with earlier findings of H04 and HS05. At low significances the spurious peaks will be dominated by shape noise, while at high significances many spurious peaks will be caused by the projection of large-scale structures. We emphasize that the latter class of peaks is in fact caused by gravitational lensing. They just do not correspond to a single mass concentration in 3-d space. These peaks are spurious peaks only in the sense of a galaxy cluster search.

Fourth, negative peaks are relatively rare. The two effects leading to this result are best understood in terms of the filter function $U$ that is related to Schirmer's $Q_{\mathrm{NFW}}$ function by Eq. (4) and acts on the surface mass density. The function $U$ has a narrow positive peak close to the origin with extended and shallow negative wings to satisfy the condition that $U$ has zero total weight. The comparably low number density of $-M_{\text {ap }}$ peaks is then caused by (1) the shallowness of negative wings, which will limit the peak strengths of negative peaks; and (2) by $U$ acting as a bandpass filter for structures with the same size as the characteristic filter scale. The large extent of the negative wings will make negative peaks more extended than positive peaks, naturally leaving less space for other peaks.

By associating peaks found in different filter scales with each other, we can also examine whether the number of filter scales $n_{\mathrm{f}}$ that a peak is detected in says something about the correspondence of the lensing signal to a dark matter halo. Figure 5 shows the number density of peaks detected in the ray-tracing simulations as a function of $n_{\mathrm{f}}$. The color/line-style coding is the same as in Fig. 4. One clearly sees that the number density of lensing peaks associated with a dark matter halo is virtually independent of the number of filter scales the $M_{\text {ap }}$ peak appears in. Real dark matter halos show up as often in only 3 filter scales as they do in 9 filter scales.

The behavior of spurious peaks is very different. Their number falls off monotonically as a function of $n_{\mathrm{f}}$, with an exception in the last bin, i.e., the peaks that are detected in all filter scales. This dependence can be used to impose a selection criterion to decrease the contamination of shear selected clusters with spurious lensing peaks by requiring that a $M_{\text {ap }}$ peak must occur in at least a given number of filter scales. However, as stated above, this will always exclude a number of real clusters as well. As

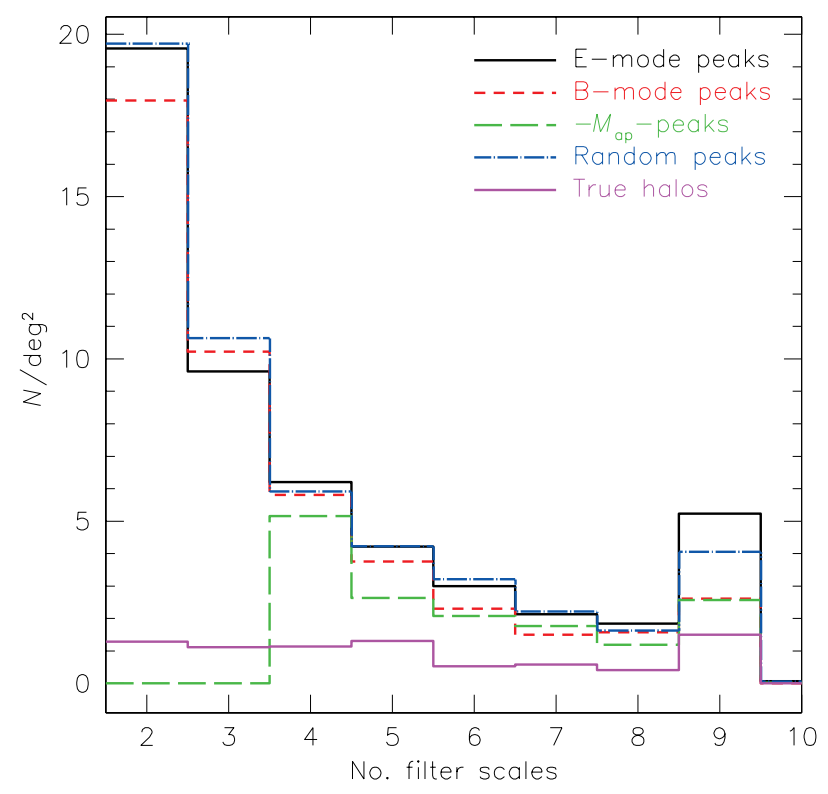

Fig. 5. Number density of lensing peaks in dependence on the number of filter scales in which the peak is detected. The color/line-style coding is the same as in Fig. 4. Dark matter halo peaks show virtually no dependence on the number of filter scales, while the number density of spurious peaks sharply declines with a requirement on the minimum number of filter scales.

a compromise between efficiency and completeness, we imposed the condition that $n_{\mathrm{f}} \geq 3$ in the XFS.

We now briefly compare these results to the ones obtained with the Maturi filter on the same catalogs. This filter can potentially result in fewer spurious peaks and increase the SNR of real clusters, thereby increasing the number of real cluster detections. We find that the results of the Schirmer and Maturi filters are entirely consistent for our survey parameters. The number of $M_{\text {ap }}$ peaks associated to dark matter halos within the 2'.15 matching radius (316) is, contrary to expectations, slightly smaller than for the Schirmer filter (325). The difference might well be due to a lower contamination with spurious peaks at high significances where the contamination with LSS projections should be suppressed by the Maturi filter. The observed peak offsets from the dark matter halo positions are consistent with the those of the Schirmer filter. We compared the peak significances of $M_{\text {ap }}$ peaks related to dark matter halos computed with both filters and find $\left\langle S\left(Q_{\mathrm{LSS}}\right)-S\left(Q_{\mathrm{NFW}}\right)\right\rangle=-0.06 \pm 0.27$, i.e., the peak significances are consistent with each other, non-significantly favoring the Schirmer filter. Again, this small difference might be due to the relative suppression of highly significant projections of the LSS. These findings agree with Maturi et al. (2007) who compare the performance of their filter with the cluster search by Schirmer et al. (2007) on the same fields. As a result of our comparison we limit the weak lensing cluster search in the XFS to the Schirmer filter that is less complex and faster to compute.

\subsection{Weak lensing catalog}

Based on the optical catalogs created with SExtractor's default convolution kernel (Sect. 4.1), we created lensing catalogs. We used the KSB algorithm (Kaiser et al. 1995) to obtain shear estimates closely following the procedure described by Erben et al. (2001). From the KSB catalogs we constructed catalogs of probable background galaxies that are reliable shear estimators by imposing the following selection criteria: objects with $S N R<2$, 
Gaussian radius $r_{\mathrm{g}}<0$. '33 or $r_{\mathrm{g}}>1$ '.' 19 , or corrected ellipticity $\varepsilon>1.0$ were deleted from the catalog. We also deleted objects whose pre-seeing shear polarizability tensor $P^{\gamma}$ has a negative trace, and bright galaxies with $R<21$ mag.

Table 1 gives the effective (unmasked) area of all XFS fields used for the weak lensing cluster search, as well as their number density of galaxies in the weak lensing catalogs. The total area used for weak lensing is $6.4 \mathrm{sq}$. deg. The number density of background galaxies averaged over this area is $14.1 \mathrm{arcmin}^{-2}$.

The aperture mass statistic with the Schirmer filter function was estimated from the resulting catalogs on the filter scales listed in Table 2. No weighting of individual galaxies was done. Peaks in the $M_{\text {ap }}$-maps were detected as described in the previous section. The final catalog lists all aperture mass peaks with maximum $S N R \geq 5$, or with maximum $S N R \geq 3$ if the peak has an X-ray or matched-filter counterpart, or was previously reported as cluster (candidate) in the literature within 2 '.15 radius from the lensing position. All shear peaks must be present in at least 3 filter scales.

In the following we give comments on some specific fields.

- T Leo - No association could be found for the $4 M_{\text {ap }}$ peaks with $\sigma_{\max }>3$ and $n_{\mathrm{f}}>2$ in this field. Thus, none of these peaks are included in our catalog.

- Field 864-1 - None of the $5 M_{\text {ap }}$ peaks with $\sigma_{\max }>3$ and $n_{\mathrm{f}}>2$ could be associated with X-ray or matched-filter clusters. These peaks are not included in our catalog.

- Field 864-9 - This field contains one of the three weaklensing selected cluster candidates that was previously identified in the literature as a cluster candidate but not found by the matched filter or in X-ray. The candidate BLOX J1343.5-0022.8 is outside the FOV of XMMNewton. The cluster candidate is very elongated and might for this reason be missed by the matched filter pipeline.

- LBQS 2212-1759 - This field contains the remaining two weak-lensing cluster candidates that match only clusters previously reported in the literature, but were found with neither the matched filter nor the X-ray survey. Both cluster candidates match optically selected clusters from $\mathrm{O} 07$, see also Sect. 4.2. Gavazzi \& Soucail (2007) have performed a weak lensing cluster search on this field as part of the CFHTLS Deep fields and have not found any convergence peak in the FOV of WFI above their detection threshold of $3.5 \sigma$.

We selected a total of 31 cluster candidates using the aperture mass method on 23 WFI fields. The full catalog of shear selected cluster candidates is available in electronic format at the CDS. There we list the position of the $M_{\text {ap }}$ peaks, the significance of their detection, the number of filter scales a cluster was detected in, and the filter scale in which the SNR of a peak was maximized. This catalog contains 4 of the 5 clusters that were primary XMM-Newton targets in the XFS fields. The fifth cluster, MKW 9, is at redshift $z=0.04$, too low to be detectable with weak lensing.

On average we find 1.3 weak lensing cluster candidates per XFS field. Most $M_{\text {ap }}$ peaks do not correspond to a cluster candidate. This is to be expected from the results of our ray-tracing simulations and illustrated by Figs. 4 and 5. The two fields containing only unmatched $M_{\text {ap }}$ peaks are statistically expected. The absence of weak lensing peaks in these fields does not hint at problems with their lensing catalogs.

\section{Summary and discussion}

In this work we have selected galaxy cluster candidates independently with three different methods: optical matched filter algorithm, extended X-ray emission, and the shear signal induced by massive foreground structures.

We found a total of 155 cluster candidates in 23 WFI fields, or 24.2 cluster candidates per square degree. Most cluster candidates were found with the optical matched filter (116), followed by X-ray emission (59). As was previously shown (H04; HS05) and confirmed by our lensing simulations using ray-tracing simulations (Sect. 5.1), the efficiency of weak lensing for cluster selection is relatively low. To avoid being dominated by spurious weak lensing signals, we limited the catalog of weak lensing selected cluster candidates to those that have an optical or X-ray counterpart, either found in our own survey or previously reported in the literature. We found significant lensing signals for 31 cluster candidates, of which 12 are previously known cluster (candidates). Eleven of the weak lensing selected clusters were detected with both the matched filter and X-ray emission, excluding A 1882 that is not part of our X-ray catalog; 6 of these are previously unknown cluster candidates.

Comparing the redshift estimates of the Postman matchedfilter method to spectroscopically measured redshifts or other photometric estimates, we find that these work surprisingly well. The mean difference in redshifts $\left\langle\Delta z /\left(1+z_{\text {true }}\right)\right\rangle$ is marginally significant only for the spectroscopic sample with $-0.06 \pm 0.05$, and consistent with zero if we also trust the redshifts of the photometric sample that gives $\left\langle\Delta z /\left(1+z_{\text {true }}\right)\right\rangle=0.06 \pm 0.07$. Considering that we use only one passband to derive the redshift of matched-filter clusters, this result compares favorably to what is achievable with more colors. For example, Goto et al. (2002) report a mean $\Delta z$ for their "cut-and-enhance" method of 0.02 using four colors, but only after outliers with $\Delta z>0.1$ have been rejected. However, the matched filter estimated redshifts come with a significant bias, which puts higher redshift cluster at redshifts that are typically too low by $\Delta z=0.1 \ldots 0.2$.

We described in detail how we developed the selection criteria for our weak lensing sample using ray-tracing simulations. We find that - at least for the comparatively low number densities and peak significances we are dealing with - the dominant source of noise is the shape noise of the background population and not projections of the large-scale structure. Our raytracing simulations suggest that the contamination with projections of the large-scale structure becomes more important at higher significances. However, the area covered by the XFS is comparatively small and the absolute number of highly significant $M_{\text {ap }}$ peaks is consequently small. The $M_{\text {ap }}$ kernel developed by Maturi et al. (2005) to minimize the effect of largescale structure on weak lensing cluster searched thus could not perform better than the filter function proposed by Schirmer et al. (2007) used in this work. It is sensible to assume that the Maturi filter will perform better on deeper surveys (Pace et al. 2007) for two reasons: (1) the higher number density of FBG will reduce the white noise component and (2) a deeper survey will probe a larger volume and hence increase the contribution of LSS projections.

The matching radius of $2 ! 15$ we deduced from our raytracing simulations is considerably smaller than the $3^{\prime}$ employed by HS05. Considering that HS05 used a higher number density of FBG with an ellipticity dispersion lower by a factor of 1.6, even their already low efficiency estimates for weak lensing cluster searches still seem to be too optimistic. 
Table A.1. Information about X-ray imaging in the XFS.

\begin{tabular}{|c|c|c|c|c|c|}
\hline \multirow{2}{*}{$\begin{array}{l}\text { Field } \\
\text { BPM } 16274\end{array}$} & \multirow{2}{*}{$\begin{array}{l}\text { Obs. ID } \\
0125320401\end{array}$} & \multirow{2}{*}{$\begin{array}{c}T_{\text {exp }} / \mathrm{s} \\
33728\end{array}$} & \multicolumn{3}{|c|}{ Camera settings } \\
\hline & & & EPN EFF & MOS1 FF & MOS2 FF \\
\hline & 0125320501 & 7845 & EPN FF & MOS1 FF & MOS2 FF \\
\hline & 0133120301 & 12022 & EPN FF & MOS1 FF & MOS2 FF \\
\hline & 0133120401 & 13707 & EPN FF & MOS1 FF & MOS2 FF \\
\hline & 0125320701 & 45951 & EPN FF & MOS1 FF & MOS2 FF \\
\hline & 0153950101 & 5156 & EPN FF & MOS1 FF & MOS2 FF \\
\hline CFRS 3h & 0041170101 & 51724 & EPN EFF & MOS1 FF & MOS2 FF \\
\hline RX J0505.3-2849 & 0111160201 & 49616 & EPN EFF & MOS1 FF & MOS2 FF \\
\hline RBS 0864 & 0108670101 & 56459 & EPN FF & MOS1 FF & MOS2 FF \\
\hline QSO B0130-403 & 0112630201 & 37870 & EPN FF & MOS1 FF & MOS2 FF \\
\hline BR 1033-0327 & 0150870401 & 31418 & EPN FF & MOS1 FF & MOS2 FF \\
\hline SDSS J104433.04-012502.2 & 0125300101 & 62310 & EPN FF & MOS1 FF & MOS2 FF \\
\hline MS1054.4-0321 & 0094800101 & 41021 & EPN FF & MOS1 FF & MOS2 FF \\
\hline HE 1104-1805 & 0112630101 & 36428 & EPN FF & MOS1 FF & MOS2 FF \\
\hline PG 1115+080 & 0082340101 & 63358 & EPN FF & MOS1 FF & MOS2 FF \\
\hline & 0203560201 & 86649 & EPN FF & MOS1 FF & MOS2 FF \\
\hline & 0203560401 & 86515 & EPN FF & MOS1 FF & MOS2 FF \\
\hline CD -3307795 & 0112880101 & 29921 & EPN FF & MOS1 FF & MOS2 FF \\
\hline T Leo & 0111970701 & 12866 & EPN FF & MOS1 SW3 & MOS2 SW2 \\
\hline IRAS $12112+0305$ & 0081340801 & 23206 & EPN FF & MOS1 FF & MOS2 FF \\
\hline LBQS $1228+1116$ & 0145800101 & 107002 & EPN FF & MOS1 FF & MOS2 FF \\
\hline NGC 4666 & 0110980201 & 58237 & EPN EFF & MOS1 FF & MOS2 FF \\
\hline QSO B1246-057 & 0060370201 & 41273 & EPN FF & MOS1 FF & MOS2 FF \\
\hline Field $864-1$ & 0111281001 & 10377 & EPN EFF & MOS1 FF & MOS2 FF \\
\hline Field $864-2$ & 0111282401 & 7077 & EPN EFF & MOS1 FF & MOS2 FF \\
\hline Field 864-4 & 0111281301 & 14541 & EPN EFF & MOS1 FF & MOS2 FF \\
\hline Field $864-5$ & 0111281401 & 8643 & EPN EFF & MOS1 FF & MOS2 FF \\
\hline Field 864-6 & 0111281501 & 8650 & EPN EFF & MOS1 FF & MOS2 FF \\
\hline Field 864-9 & 0111282501 & 8623 & EPN EFF & MOS1 FF & MOS2 FF \\
\hline A 1882 & 0145480101 & 23567 & EPN FF & MOS1 FF & MOS2 FF \\
\hline MKW 9 & 0091140401 & 45414 & EPN EFF & MOS1 FF & MOS2 FF \\
\hline LBQS 2212-1759 & 0106660101 & 60508 & EPN FF & MOS1 FF & MOS2 FF \\
\hline & 0106660201 & 53769 & EPN FF & MOS1 FF & MOS2 FF \\
\hline & 0106660401 & 35114 & - & MOS1 FF & MOS2 FF \\
\hline & 0106660501 & 11568 & EPN FF & MOS1 FF & MOS2 FF \\
\hline & 0106660601 & 110168 & EPN FF & MOS1 FF & MOS2 FF \\
\hline NGC 7252 & 0049340201 & 28359 & EPN FF & MOS1 FF & MOS2 FF \\
\hline PHL 5200 & 0100440101 & 46681 & EPN FF & MOS1 FF & MOS2 FF \\
\hline
\end{tabular}

The number density of weak-lensing selected cluster candidates is 4.8 per square degree. This is slightly lower than the number density of $M_{\text {ap }}$ peaks with a halo counterpart in the ray-tracing simulations, which is $6.1 / \mathrm{sq}$. deg. However, the average number density of background galaxies in the XFS data is only $14.1 \mathrm{arcmin}^{-2}$; this is somewhat lower than in the raytracing simulations, which had an average number density of $16.5 \mathrm{arcmin}^{-2}$. Whether the difference in cluster counts can really be attributed to the difference in number density should be checked by adjusting the simulation parameters to match the XFS observations better. The trend to slightly lower number densities is also present if we select only $M_{\text {ap }}$ peaks with a higher $S N R \geq 4$. The XFS contains 11 of these highly significant peaks associated with a matched filter or X-ray cluster candidate. This corresponds to $1.7 / \mathrm{sq}$. deg, compared to $2.3 / \mathrm{sq}$. deg in the raytracing simulations. These high significance peaks include all 4 clusters that were primary XMM-Newton targets at redshifts accessible by weak lensing. These 4 clusters alone contribute a number density of $0.6 / \mathrm{sq}$. deg.

With the cluster sample presented here we have built a solid foundation for studying possible selection effects in either method. This will be the subject of a follow-up paper (Dietrich et al., in preparation).
Acknowledgements. We thank the anonymous referee for many comments that helped to improve the clarity of this paper. This work was supported by the German Ministry for Science and Education (BMBF) through DESY under the project 05AE2PDA/8, by the Deutsche Forschungsgemeinschaft under the project SCHN 342/3-1, by the German DLR under contract number 50OX0201, and by the Priority Program 1177 of the Deutsche Forschungsgemeinschaft.

\section{Appendix A: X-ray observations}

Table A.1 gives a summary of the EPIC X-ray observations contributing to the XFS data used in this work. The table gives for each field: in Col. 1 the field name; in Col. 2 the XMM-Newton observation ID; in Col. 3 the nominal exposure time in seconds; in Cols. 4-6 the settings for each of the cameras. Here (E)FF indicates (extended) full frame readout, LW large-window mode, and SW small-window mode. These cameras and their settings are described in detail in Ehle et al. (2004). For some fields additional observations were available, but these were discarded mainly due to unsuitable camera settings.

"Field 864 " is a mosaic of $3 \times 3$ XMM-Newton observations. The name was assigned by the principal investigator. Fields 864-2, 4, 5, 6 are listed in Table A.1 although they are not covered by our WFI observations. The reason is that these data overlap with the Fields 864-6, and 9, which are XFS survey fields. 


\section{References}

Abell, G. O. 1958, ApJS, 3, 211

Bartelmann, M. 1996, A\&A, 313, 697

Bartelmann, M., \& Schneider, P. 2001, Phys. Rep., 340, 291

Benoist, C., da Costa, L., Jørgensen, H. E., et al. 2002, A\&A, 394, 1

Bertin, E., \& Arnouts, S. 1996, A\&AS, 117, 393

Borgani, S., \& Guzzo, L. 2001, Nature, 409, 39

Dietrich, J. P., Schneider, P., Clowe, D., Romano-Díaz, E., \& Kerp, J. 2005, A\&A, 440, 453

Dietrich, J. P., Miralles, J.-M., Olsen, L. F., et al. 2006, A\&A, 449, 837

Ehle, M., Breitfellner, M., González Riestra, R., et al. 2004, XMM-Newton Users' Handbook

Eke, V. R., Cole, S., \& Frenk, C. S. 1996, MNRAS, 282, 263

Erben, T., van Waerbeke, L., Mellier, Y., et al. 2000, A\&A, 355, 23

Erben, T., Van Waerbeke, L., Bertin, E., Mellier, Y., \& Schneider, P. 2001, A\&A, 366,717

Erben, T., Miralles, J. M., Clowe, D., et al. 2003, A\&A, 410, 45

Erben, T., Schirmer, M., Dietrich, J. P., et al. 2005, Astron. Nachr., 326, 432

Gal, R. R. 2006 [arXiv: astro-ph/0601195]

Gavazzi, R., \& Soucail, G. 2007, A\&A, 462, 459

Gladders, M. D., \& Yee, H. K. C. 2000, AJ, 120, 2148

Goto, T., Sekiguchi, M., Nichol, R. C., et al. 2002, AJ, 123, 1807

Hamana, T., Takada, M., \& Yoshida, N. 2004, MNRAS, 350, 893 (H04)

Hansen, L., Olsen, L. F., \& Jørgensen, H. E. 2002, A\&A, 388, 1

Hartlap, J. 2005, Diplomarbeit, Universität Bonn, Germany

Hennawi, J. F., \& Spergel, D. N. 2005, ApJ, 624, 59 (HS05)

Henry, J. P. 2004, ApJ, 609, 603

Hetterscheidt, M., Erben, T., Schneider, P., et al. 2005, A\&A, 442, 43

Hetterscheidt, M., Simon, P., Schirmer, M., et al. 2006 [arXiv: astro-ph/0606571]

Jenkins, A., Frenk, C. S., Pearce, F. R., et al. 1998, ApJ, 499, 20
Jenkins, A., Frenk, C. S., White, S. D. M., et al. 2001, MNRAS, 321, 372

Kaiser, N., Squires, G., \& Broadhurst, T. 1995, ApJ, 449, 460

Maoli, R., Van Waerbeke, L., Mellier, Y., et al. 2001, A\&A, 368, 766

Maturi, M., Meneghetti, M., Bartelmann, M., Dolag, K., \& Moscardini, L. 2005, A\&A, 442, 851

Maturi, M., Schirmer, M., Meneghetti, M., Bartelmann, M., \& Moscardini, L. 2007, A\&A, 462, 473

Miralles, J.-M., Erben, T., Hämmerle, H., et al. 2002, A\&A, 388, 68

Mullis, C. R., Rosati, P., Lamer, G., et al. 2005, ApJ, 623, L85

Olsen, L. F., Scodeggio, M., da Costa, L., et al. 1999, A\&A, 345, 681 (099)

Olsen, L. F., Hansen, L., Jørgensen, H. E., et al. 2003, A\&A, 409, 439

Olsen, L. F., Benoist, C., Cappi, A., et al. 2007, A\&A, 461, 81 (O07)

Pace, F., Maturi, M., Meneghetti, M., et al. 2007 [arXiv: astro-ph/0702031]

Postman, M., Lubin, L. M., Gunn, J. E., et al. 1996, AJ, 111, 615

Press, W. H., \& Schechter, P. 1974, ApJ, 187, 425

Schirmer, M., Erben, T., Hetterscheidt, M., \& Schneider, P. 2007, A\&A, 462, 875

Schneider, P. 1996, MNRAS, 283, 837

Schneider, P., \& Seitz, C. 1995, A\&A, 294, 411

Soneira, R. M., \& Peebles, P. J. E. 1978, AJ, 83, 845

Stanford, S. A., Romer, A. K., Sabirli, K., et al. 2006, ApJ, 646, L13

Stetson, P. B. 2000, PASP, 112,925

Umetsu, K., \& Futamase, T. 2000, ApJ, 539, L5

Van Waerbeke, L., Mellier, Y., \& Hoekstra, H. 2005, A\&A, 429, 75

Vikhlinin, A., Voevodkin, A., Mullis, C. R., et al. 2003, ApJ, 590, 15

von der Linden, A., Erben, T., Schneider, P., \& Castander, F. J. 2006, A\&A, 454, 37

Weinberg, N. N. \& Kamionkowski, M. 2002, MNRAS, 337, 1269

Wittman, D., Dell'Antonio, I. P., Hughes, J. P., et al. 2006, ApJ, 643, 128

Wittman, D., Tyson, J. A., Margoniner, V. E., Cohen, J. G., \& Dell'Antonio, I. P. 2001, ApJ, 557, L89

Wright, C. O., \& Brainerd, T. G. 2000, ApJ, 534, 34 